\title{
Multiparametric assessment of the efficacy of voice therapy in the management of hyper functional voice disorders
}

\author{
Abstract \\ Aim of the study: The present study was aimed at investigating the efficacy of an eclectic \\ voice therapy program in the treatment of hyperfunctional voice disorders using single \\ subject time series research design and systematic documentation. \\ Objectives of the study: \\ i. To assess the voice quality objectively in individuals with hyperfunctional voice \\ disorders using the multiparametric measure dysphonia severity index (DSI) \\ ii. To measure the change in vocal performance using eclectic voice therapy approach.
}

Volume 8 Issue 2 - 2017

\author{
Goipkishore P, Pushpavathi M \\ Department of Speech Language Pathology All India Institute of \\ Speech and Hearing, India
}

Correspondence: Goipkishore P Lecturer in Speech Pathology Department of Speech Language Pathology All India Institute of Speech and Hearing Mysore, India, Tel +919036377965, Email gopiaaslp@gmail.com

Received: May 25, 2017| Published: July 26, 2017

Keywords: hyperfunctional voice disorder, muscle tension dysphonia, dysphonia severity index,eclectic voice therapy

Abbreviations: HFVD, hyperfunctional voice disorder; DSI, dysphonia severity index; MTD, muscle tension dysphonia; CSL, computerized speech lab; SPSS, statistical package for social secinces; VRP, voice range profile

\section{Introduction}

Hyperfunctional voice disorder (HFVD) is a dysphonic condition that is not associated with neurological, malignant, dysplastic, inflammatory, immunologic endocrine or psychiatric issues or with laryngeal trauma or injury. ${ }^{1}$ Different types and degrees of abnormal and/or excessive muscle tension in the laryngeal muscles during phonation are considered as essential features for HFVD. Several terms such as muscle tension dysphonia (MTD) and tension fatigue syndrome has been used synonymously in the literature. Multiple factors such as vocal misuse or abuse subsequent to high vocal demands pertaining to different occupations, age, prolonged usage of voice, lifestyles, socioeconomic status, self correction \& regulation of voice using sensory feedback, compromised breath support, persistent upper respiratory tract infections, gastroesophageal reflex disorder etc have been reported to cause this condition. Professional voice users with high vocal demands such as teachers, singers, politicians, cheer leaders etc. are prone to develop hyperfunctional voice disorders. Among this category, the prevalence of voice problems is more in teachers compared to the other professions. ${ }^{2}$ Prevalence of voice problems among teachers varied from $20 \%$ to $75 \%{ }^{3-5}$ Hyperfuncional voice disorders were reported to be in $32.7 \%$ of the teachers. ${ }^{6}$ Sebastian et al. (2012) reported $9 \%$ of the teachers to have voice problems and mentioned that the allergic rhinitis, laryngitis, sinusitis, gastroesophageal reflux, minimal sensori neural hearing loss and hypothyroidism were the major medical factors associated with those $9 \%$ teachers.

The prominent clinical characteristics in HFVD include elevated muscular tension in the laryngeal and perilaryngeal musculature, altered vertical laryngeal position, pain and tightness in the anterior neck region, larynx, and shoulder regions. ${ }^{8,9}$ The voice characteristics in HFVD depend on the type of resulting glottic closure. Based on the type of glottic closure and the specific group of muscles involved, the HFVD was further classified as adductor hyperfunction and abductor hyperfunction. Hence, based on the severity and sub type of HFVD, the individuals with HFVD demonstrate several voice quality types including hoarseness, pressed voice, glottal fry, breathiness, diplophonia, high-pitched falsetto, as well as voice and pitch breaks 8; Roy, 2004).

Considering the multifactorial origin of this condition, several direct and indirect voice therapy approaches targeting various facets of voice production have been attempted and researched. For instance, Mathieson et al., ${ }^{10}$ used laryngeal manual therapy with the aim of relaxing laryngeal musculature in individuals with MTD and reported positive outcomes on the acoustic measures, palpatory evaluation and on vocal tract discomfort scale. Van Lierde et al., ${ }^{11}$ compared circumlaryngeal manual therapy and vocalizations with abdominal breath support in the treatment of individuals with MTD. Results indicated statistically significant improvement with circumlaryngeal manual therapy on acoustic and aerodynamic outcome measures.

To summarize, there have been attempts made to promote evidence based practice in the treatment of voice disorders by conducting treatment efficacy studies. However, the outcome of the studies was confined due to the factors such as the absence of control group, limited duration of intervention, limited sample size, only pre-post measures, or a combination of any of these. Although most of the studies have attempted to find the efficacy of single treatment approaches, those studies which considered eclectic approach reported to have better clinical outcome. Nevertheless, very few attempts have been made to investigate the efficacy of the eclectic voice therapy programmes in the treatment of hyperfunctional voice disorder. It is essential to investigate the application of an eclectic / combinatory approach in the management of hyperfunctional voice disorders. Further, the outcomes of these studies must be verified using sensitive parameters and controlled research designs.

\section{Methods}

\section{Participants}

Forty four individuals including eleven males and thirty three females in the age range of 15 to 64years (average age of 33.4years) who fulfilled the following criterion were included into the study. 
1. Individuals with complaint of vocal fatigue 2 . Vocal strain and hoarseness of voice on analysis by a SLP experienced with treating voice disorders. 3. Individuals with changes to laryngeal mechanism due to vocal hyperfunction such as glottal chink, hypertrophy of ventricular folds, excessive supraglottic contractions with or without minimal alterations to vocal fold mucosa such as early vocal nodules, irregular edge of the vocal folds etc as observed on videostroboscopy. However, the individuals with mass occupying lesions such as vocal polyp, vocal cysts, contact ulcers etc were excluded from the study.

The participants were included based on the evaluation by the team of an Otolaryngologist and Speech Language Pathologist using detailed case history, palpatory evaluation and finally through video laryngoscopy with focus on identifying the glottic and supraglottic variations. The classification given by Morrison and Rammage was used to identify the participants with vocal hyperfunctional conditions into one of the types of muscle tension dysphonia such as MTD type I, type II a, type II b etc. In the current study $60 \%$ (12 out of 20 ) of the participants were professional voice users, with majority of them being teachers. The details of the participants are given under Table 1.

The study required participants to attend 16 sessions of eclectic voice therapy and one follow up session with a gap of one month. Although a total of 44 individuals were enrolled initially, due to personal and professional related reasons several of the participants dropped out following one to three voice therapy sessions. Finally 20 Individuals were able to attend a minimum of six sessions of voice therapy. The number of therapy sessions attended by the participants varied ranging from minimum of six to fourteen sessions. Among the 20 participants, three of them attended 14 sessions, five of them attended 10 sessions, six of them attended eight sessions and remaining six attended only six sessions of voice therapy. A signed consent form including the details about the aim and method of the current research study was obtained from the participants.

\section{Procedure}

Voice therapy procedure: The voice therapy procedure included four stages.

i. Working on improving participant's vocal hygiene awareness. The initial two sessions for each participant were exclusively spent on sensitizing the participant regarding the vocal pathology, vocal hygiene, and steps to prevent further vocal damage such as increased vocal hydration, reduced throat clearing, avoiding speaking over competing noise etc.

ii. Secondly facilitating coordination between the respiratory and phonatory systems with the aim of developing vocal efficiency

iii. Thirdly reducing excessive muscle tension in the laryngeal and perilaryngeal region using Laryngeal Manual Therapy

iv. Focus on improving the quality of voice by promoting forward focus through resonant voice therapy.

Objective measurement of dysphonia severity: Dysphonia severity index (DSI) is a multiparametric approach that was designed to establish an objective and quantitative correlate of the perceived vocal quality. ${ }^{12}$ The DSI is weighted combination of selected set of voice measurements such as maximum phonation time (MPT in seconds), highest frequency (F0 high in Hz), lowest intensity (I-low in $\mathrm{dB}$ ), and jitter $(\%)$. The DSI is constructed using the equation, DSI $=0.13 \mathrm{x}$ MPT(s) $+0.0053 \times$ Fo-high $(\mathrm{Hz})-0.26$ x I-low $(\mathrm{dB})-1.18 \times$ jitter $(\%)+12.4$. The DSI of perceptually normal voice equals to +5 and for severely dysphonic voices -5 . DSI was used to document the severity of dysphonia objectively.

For constructing the DSI, all these parameters were extracted from the Computerized Speech Lab(CSL) (Kay Elemetrics Corp., Lincoln Park, New Jersy; Model 4500). To obtain maximum phonation time, participant was instructed to sit in a comfortable posture and sustain the vowel /a/ at habitual pitch and loudness. The procedure was modeled by the experimenter and the participant was also visually encouraged during his turn of vowel prolongation. The length of the sustained vowel (in seconds) was measured by placing two cursors at either end of the voicing sample on the recording window of Computerized Speech Lab (CSL). Initially two trials of MPT were obtained followed by three test recordings of MPT and the best (longest duration) of the three test recordings was retained for further analysis and descriptions.

The highest frequency and the lowest intensity were measured with the aid ofvoice range profile(VRP) module of CSL (4500). The recordings of Fo-high and I-low were obtained with the instrument in VRP mode and with a table mounted microphone kept at a constant distance of $10 \mathrm{~cm}$ from the mouth. For obtaining highest frequency, the participant was instructed to glide the vowel /a/. The participant was further instructed and encouraged to use the visual feedback from the display on the computer screen to produce the best possible highest frequency. Initially two trials were obtained followed by three test recordings and the best of the three highest frequencies on the test recordings was considered for further analysis. For obtaining lowest intensity (I-low), the participants were instructed to produce the vowel /a/ as soft as possible. The recording was be done using the CSL in VRP mode and with the device switchcraft (to obtain the absolute intensity measures by nullifying the effect of pre-amplification) connected to the microphone. Three test recordings were obtained followed by two trials and the lowest of the three lowest intensities on test recordings was recorded and considered for construction of DSI.

Jitter (\%) was determined with the aid of Multi Dimensional Voice Program (MDVP) module of CSL 4500. The participant was instructed to sit in a comfortable posture and sustain the vowel /a/ at habitual pitch and loudness to a table mounted microphone kept at a constant distance of $10 \mathrm{~cm}$ from participant's mouth and connected to the hardware of CSL. The recording was done at $44000 \mathrm{~Hz}$ sampling rate in the window length of five seconds. A mid vowel segment of three seconds duration was selected for analysis by avoiding the initial and final one second segments. The jitter (\%) obtained on analysis was used for further calculations.

With the obtained values, the DSI was then constructed by entering the constituent values in the equation DSI $=0.13 \mathrm{x}$ MPT $(\mathrm{s})+0.0053$ x Fo-high $(\mathrm{Hz})-0.26$ x I-low $(\mathrm{dB})-1.18$ x jitter $(\%)+12.4$. The DSI values were obtained at the baseline (before commencing voice therapy) and following the completion of every two sessions of voice therapy.

\section{Results}

The results indicated that the DSI value varied from negative to positive across the sessions. The negative DSI value indicates more severe voice disorder, whereas, positive DSI value indicate good/improved voice quality. It may be observed from the Figure 1 that the each of the participant differed in their baseline DSI value indicating the differences in their baseline severity of voice disorder. For instance, participants $\mathrm{O}, \mathrm{T}$ and $\mathrm{J}$ had baseline DSI value below zero indicating more severe voice disorder than compared to $\mathrm{E}$, I and S participants with relatively higher baseline DSI value. 
Table I Details of the participants included in the study

\begin{tabular}{|c|c|c|c|c|}
\hline Participants & Age Years) & Gender & Profession & Stroboscopic Findings \\
\hline A & 38 & $\mathrm{~F}$ & Teacher & Vocal Nodules \\
\hline B & 32 & $\mathrm{~F}$ & Teacher & Glottic Chink with MTD \\
\hline C & 31 & $\mathrm{~F}$ & Govt. Officer & Bilateral early Vocal Nodules \\
\hline $\mathrm{D}$ & 44 & $\mathrm{~F}$ & Home Maker & Bilateral early Vocal Nodules \\
\hline $\mathrm{E}$ & 32 & $\mathrm{~F}$ & Teacher & Early Nodules with MTD III \\
\hline $\mathrm{F}$ & 15 & $M$ & Student & Early Nodules with MTD II b \\
\hline G & 23 & $\mathrm{~F}$ & Speech Pathology Student & MTD type I \\
\hline $\mathrm{H}$ & 34 & $\mathrm{~F}$ & Rekhi Practitioner & Glottic chink with MTD type 3 \\
\hline I & 35 & $\mathrm{~F}$ & Home Maker & MTD II \\
\hline J & 44 & $M$ & Teacher & Glottic Chink \\
\hline K & 24 & $F$ & Student & Glottic Chink \\
\hline L & 28 & $\mathrm{~F}$ & Music Student & MTD with early Vocal Nodules \\
\hline M & 42 & $\mathrm{~F}$ & Teacher & Glottic chink with MTD type III \\
\hline N & 42 & $\mathrm{~F}$ & Singer / Home Maker & MTD \\
\hline O & 38 & $\mathrm{~F}$ & Teacher & MTD type II with early Vocal Nodules \\
\hline $\mathrm{P}$ & 47 & $\mathrm{~F}$ & Bank Manager & MTD \\
\hline Q & 18 & $\mathrm{~F}$ & Student & MTD with Glottic Chink \\
\hline $\mathrm{R}$ & 27 & $\mathrm{~F}$ & Home maker & Post-Surgical for Right Vocal fold Polyp \\
\hline S & 15 & M & Student & Bilateral Vocal Nodules \\
\hline $\mathrm{T}$ & 64 & M & Retired Employee & $\begin{array}{l}\text { Spindle Shaped Glottic Closure with false VF } \\
\text { Hypertrophy with MTD II }\end{array}$ \\
\hline
\end{tabular}

Table 2 DSI value of participants across the sessions

\begin{tabular}{|c|c|c|c|c|c|c|c|c|}
\hline Participants & OI & O2 & O3 & 04 & 05 & 06 & 07 & 08 \\
\hline A & -2.32 & 3.29 & 3.46 & 2.63 & 3.42 & 4.12 & 2.76 & 3.8 \\
\hline B & 1.7 & -0.83 & 2.75 & 3.97 & 4.33 & & & \\
\hline C & 4.95 & 3.14 & 2.73 & 6.11 & 6.44 & 6.15 & 6.6 & 7.12 \\
\hline D & 0.33 & -0.45 & $-0.4 I$ & 2.21 & 3.5 & 4.3 & 3.22 & 3.84 \\
\hline$E$ & 3.03 & 2.73 & 4.55 & 4.94 & 4.86 & & & \\
\hline $\mathrm{F}^{*}$ & -1.34 & 2.99 & 3.42 & 2.83 & & & & \\
\hline G & 3.24 & 3.67 & 1.55 & 3.18 & & & & \\
\hline $\mathrm{H}$ & -0.08 & 1.76 & 2.59 & 2.52 & 1.81 & 3.65 & & \\
\hline I & 3.97 & 5.69 & 4.48 & 5.65 & 5.72 & & & \\
\hline$J^{*}$ & -2.57 & -2.59 & -1.62 & -0.47 & & & & \\
\hline K & 3.18 & 3.54 & 3.77 & 4.42 & 4.32 & & & \\
\hline L & 1.5 & I & 3.39 & 4.04 & 4.51 & & & \\
\hline$M$ & -1.08 & 3.86 & 2.91 & 3.89 & 3.83 & 4.37 & & \\
\hline $\mathrm{N}$ & $\mathrm{I} .4$ & 3.54 & 2.82 & 3.99 & 3.9 & 3.2 & & \\
\hline O & 2.34 & -3.46 & 4.17 & 5.3 & 4.16 & 3.53 & & \\
\hline$P$ & 0.49 & 1.82 & 2.2 & 3.55 & 3.74 & & & \\
\hline Q & 2.84 & 3.84 & 3.14 & 4.86 & & & & \\
\hline $\mathrm{R}$ & 0.02 & 0.61 & 1.8 & 2.76 & & & & \\
\hline $\mathrm{S}^{*}$ & I.53 & 3.48 & 6.13 & 6.25 & & & & \\
\hline $\mathrm{T}$ & -4.27 & 4.35 & 2.96 & 1.92 & 5.55 & 5.83 & & \\
\hline
\end{tabular}

*Male participants.

Note: OI: Baseline; O2:Two Sessions of Therapy; O3: Four Sessions of Therapy; O4: Six Sessions of Therapy; O5: Eight Sessions of Therapy; O6:Ten Sessions of Therapy; O7:Twelve Sessions of Therapy; O8: Fourteen Sessions of Therapy.

Table 3 Mean and Standard Deviation of DSI value across the sessions

\begin{tabular}{llllll}
\hline Observations & M & SD & Median & Min - Max & Range \\
\hline OI & 1.24 & 2.36 & 1.49 & -4.27 to 4.95 & 9.22 \\
O2 & 2.24 & 2.27 & 3.14 & -3.46 to 5.69 & 9.15 \\
O3 & 2.87 & 1.19 & 2.91 & -0.41 to 4.55 & 4.96 \\
O4 & 3.87 & 1.23 & 3.97 & 1.92 to 6.11 & 4.19 \\
\hline
\end{tabular}

Note: OI: Baseline; O2:Two Sessions of Therapy; O3: Four Sessions of Therapy; O4: Six Sessions of Therapy; M: Mean; SD: Standard Deviation

Citation: Goipkishore P, Pushpavathi M. Multiparametric assessment of the efficacy of voice therapy in the management of hyper functional voice disorders. $J$ Otolaryngol ENT Res. 2017;8(2):4 I4 420. DOI: I0.15406/joentr.2017.08.00239 
Further, two of the participants $\mathrm{C}$ and I demonstrated higher DSI value at the baseline, i.e. DSI value within the normative range reported in the earlier studies conducted on phononormic Indian adults. ${ }^{13}$ However, they were considered for the treatment based on their pathological conditions as noted on video laryngoscopy, some aspects of their perceptual voice quality such as strain / asthenia and based on the participant's complaints such as vocal fatigue.

In terms of change in DSI with therapy as depicted in Table 2, DSI value moved towards positive side with the progress in the voice therapy sessions. The poor DSI value is exhibited in the base line is - 4.27 by the participant $\mathrm{T}$ whereas after ten sessions of therapy, the value reached to 5.83. With reference to the changes in DSI between observations, a noticeable change was seen in the DSI from $\mathrm{O} 1$ to $\mathrm{O} 2$ in which seven of the 20 participants B, C, D, E, J, L, and O had shown in decrease in the DSI value. However, six out of these seven participants attained improved DSI value by the third observation $\mathrm{O} 3$ and the remaining participant ' $\mathrm{D}$ ' shown positive change at the fourth observation $\mathrm{O} 4$ i.e. after completion of six voice therapy sessions.

Majority of the participants obtained more than 3 points on DSI value by the time they got discharged or dropped out of therapy. Even participants with very severe voice problem for instance participant ' $T$ ' with DSI value of -4.27 improved to a DSI value of 5.82 over 10 sessions of voice therapy. Among the 20 participants, only one participant i.e., 'J' remained in negative DSI value even after attending six sessions of voice therapy. Although a marginal change, even this participant's DSI value moved from -2.57 at first observation to -0.47 by the fourth observation before being dropped out from therapy.

\section{Group Statistics of DSI scores}

The DSI scores evaluated from $\mathrm{O} 1$ to $\mathrm{O} 4$ reflecting the baseline and output up to six therapy sessions was considered for group statistics using Statistical Package for Social Sciences (SPSS) software 16 version. Hence, only four observations of DSI data points were included for statistical analysis. The group statistics were only performed separately for female participants as there were only 3 males and DSI scores may get affected by the gender differences.

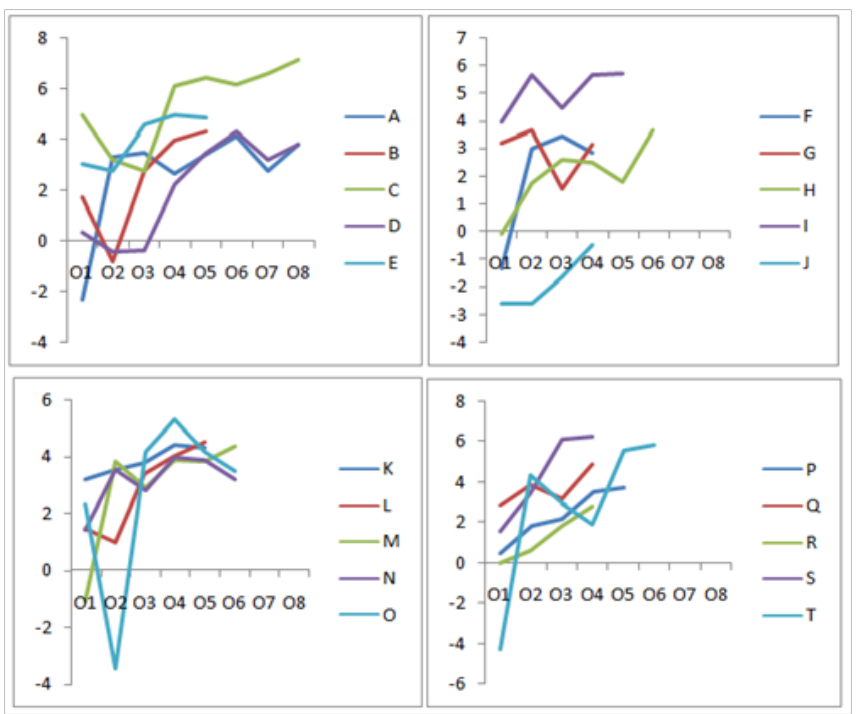

Figure I DSI value of participants across the sessions. OI: Baseline; O2: 2 Sessions of Therapy; O3: 4 Sessions of Therapy; O4: 6 Sessions of Therapy; O5: 8 Sessions of Therapy; O6: 10 Sessions of Therapy; O7 = 12 Sessions of Therapy; O8: I 4 Sessions of Therapy.

\section{Descriptive and parametric statistical test results}

Followed by the normality test, the data was subjected to parametric statistical test i.e., Repeated measures of ANOVA. The results of the descriptive statistical tests are reported in Table 3. It indicates the mean, standard deviation, median and range of DSI value of the participants at baseline and across the sessions. Table 2 shows that the DSI values were increasing from baseline observation (1.24) to the following observations and reaching 3.87 in the fourth observation indicating a positive effect of voice therapy on improving the voice characteristics. Further, the reduced standard deviation value from first observation (2.36) to fourth observation (1.23) and range also from $\mathrm{O} 1$ (9.22) to $\mathrm{O} 4$ (4.19) indicates reduced variability among the participants with respect to their voice related quality of life at post therapy phase.

The repeated measured analysis of variance (ANOVA) was conducted to evaluate the null hypothesis that there is no change in participant's DSI scores when measured before therapy (O1), during therapy $(\mathrm{O} 2, \mathrm{O} 3, \mathrm{O} 4)$ in a group of females $(\mathrm{n}=17)$ with MTD. The results of ANOVA indicated a significant therapy effect $\mathrm{F}(3,48)=7.86$, $\mathrm{p}<0.01$. Follow up comparisons indicated that statistically significant increase in DSI value is existing between $\mathrm{O} 1$ and $\mathrm{O} 4(\mathrm{p}<0.01)$ and $\mathrm{O} 3$ and $\mathrm{O} 4(\mathrm{p}<0.01)$. This result indicates that the six sessions of voice therapy can increase the DSI scores significantly in individuals with MTD.

\section{Discussion}

DSI has been considered as a robust and reliable measure and has been used frequently in the several efficacy studies. This parameter has been reported as a good correlate of perceptual dysphonia severity. In the current study this parameter was used to document the changes in voice quality across the therapy sessions in participants with hyperfunctional voice disorders. The present study hypothesized that an eclectic voice therapy program comprising of patient education along with systematic implementation of symptomatic voice therapy approaches could promote better prognosis in individuals with HFVD. In order to verify the hypothesis, 20 participants in the age range of 15-65years diagnosed with HFVD by a team consisting of a Laryngologist and a Speech Language Pathologist were considered for the study. Outcome of the therapy was documented before commencing the therapy (baseline) and following every two sessions during the therapy using the objective parameter dysphonia severity index (DSI).

Results related to the DSI revealed several points of interest. The first point of interest was the variation among the baseline DSI value across the participants. Among the twenty participants, the DSI value varied from negative to positive with lowest and highest value being -4.2 and +4.95 respectively. This indicates that the severity of voice disorder and dysphonia varied across the participants. This can be attributed to the pathological variations among the participants. Although all the participants included in the study were with hyperfunctional voice disorders, there was variation with respect to the extent and type of abnormal glottic and supraglottic activity. While some participants exhibited excessive muscular activity at the level of glottis as well as in the aryepiglottic folds and ventricular folds, others had only supraglottic hyperfunction with normal glottic closure. Further, there was variation in terms of participant's vocal fold edge. While some had normal vocal fold edge, eight of the participants had early vocal nodules. This difference in the type and severity of vocal pathologies across the participants explains the variation in the baseline DSI value across participants. 
The baseline variations in DSI can also be attributed to the variations in its constituent parameter maximum phonation duration. The maximum phonation duration of an individual reflects the breath support i.e. respiratory capacity and respiratory-phonatory control. There were variations in the pattern of breathing and respiratoryphonatory control among the participants. The participants breathing patterns included predominantly thoracic with clavicular and abdominal in some of them. These differences would have lead to the variations in the maximum phonation duration and in turn the overall DSI value of the participants at baseline. These findings are in consonance with that of Van Lierde et al. ${ }^{14}$ who reported a mean DSI value of -1 with value ranging from -14 to +2.3 and a standard deviation of 4.5. Even in the phononormic individuals DSI was shown to vary widely from 3.79 to $6.25 .{ }^{13}$

The second point of interest that was observed was the variation in the DSI value across the voice therapy sessions. Although there were individual variations in terms of the magnitude of improvement observed, the DSI value of all the participants moved in positive direction indicating improved voice with proposed EVT program. De Bodt et al., ${ }^{15}$ stated that DSI is a sensitive multi parametric measure even $5 \%$ change indicates improvement in voice quality. The improvement in the overall DSI value can be attributed to the improvement in the respiratory capacity, respiratory-phonatory control, the relaxed laryngeal frame work and improved vocal stability. While improved respiratory-phonatory control enhances the maximum phonation duration, relaxed laryngeal musculature and improved vocal stability enhance the highest frequency and reduced jitter \% respectively, hence improving the overall the DSI value.

Similar enhancement in DSI value was reported by Van Lierde ${ }^{16}$ using combination of classical approaches such as chant talk, open mouth approach, along with establishing abdominal pattern of breathing and repeated laryngeal manipulation. They also reported improvement in acoustic measures higher frequency, fundamental frequency, and perturbations, with jitter and shimmer reaching normalcy in almost all the participants. Similarly, Amir, Wingate et al., ${ }^{17,18}$ also reported significant improvement in voice quality with eclectic voice therapy. Roy, Leeper et al.,,19 also reported marked improvement in the acoustic measures even with a single treatment approach manual circumlaryngeal therapy targeting laryngeal muscle relaxation.

The improvement observed in the DSI indicates the change in voice quality. This can be attributed to many factors. According to Aronson, ${ }^{8}$ the chronic posture of the larynx in an elevated position results in cramping and stiffness of the hyolaryngeal musculature and a direct therapy approach such as circum laryngeal manual therapy directly reduces laryngeal tension thus resulting in an immediate improvement in voice. In the current study, pre-therapeutic palpatory evaluation revealed all the participants having elevated tension in the strenocliedomastoid muscles and supralayngeal musculature involving mylohyoid and geniohyoid. In addition majority of the participants demonstrated elevated vertical laryngeal height at rest and on phonation indicating the typical features of muscle tension dysphonia. Laryngeal manual therapy (LMT) was used in the current study for the purpose of relaxation of laryngeal and perilaryngeal musculature. As the steps used in LMT such as massing sternocliedomastoid muscles and kneading the supralaryngeal musculature are similar to the described by Aronson, ${ }^{8}$ the improvement in the voice quality as observed on improved DSI value could be attributed to the altered laryngeal framework following LMT.
According to Pettersen et al., ${ }^{20,21}$ the individual vocal tract structures have influence on tension and movements of each other and hence correction of tension in one of the vocal tract muscle group have positive influence on other parts of the vocal tract. This explains the influence of massaging the major muscle of the neck sternocliedomastoid on the overall relaxation of the laryngeal framework. Further, improvement in the body posture and change in the respiratory patterns were reported to contribute to the decreased tension in the suprahyoid area, which in turn relaxes the larynx and lowers in vertical position. ${ }^{22}$ Therefore, using an eclectic voice therapy program with combination of respiratory, phonatory and resonatory exercises in the current study could also have facilitated laryngeal relaxation by developing balance across these sub systems of voice production.

The third point can be the change of DSI with progression in therapy sessions. The increase in the mean DSI value was observed from $\mathrm{O} 1$ (baseline) to $\mathrm{O} 2, \mathrm{O} 1$ to $\mathrm{O} 3$ and $\mathrm{O} 1$ to $\mathrm{O} 4$, and the differences were statistically significant from $\mathrm{O} 1$ to $\mathrm{O} 3$ and $\mathrm{O} 4$. However, when DSI value of the individual participants were considered, the participants B, D, E, J, L, O, shown a reverse trend in which the DSI value was deteriorated following two sessions of therapy (at $\mathrm{O} 2$ ). This indicates the deterioration in voice quality initially with voice therapy which moved positively only after their third therapy session i.e. at third, fourth and further observations. This could be attributed to the sensitivity that the participants gained following vocal hygiene education in the first two sessions. During these two sessions, the participants were educated on vocal hygiene aspects such as the ill effects of producing effortful voice, phonating on end of the breath, use of breathy attack for initiation of phonation. Therefore, it might be possible that the participants were not using the abnormal compensations, thus reducing the maximum phonation time, highest f0 and thus reducing the overall DSI value. Further, increase in the DSI value at third observation could due to the introduction of systematic implementation of laryngeal manual therapy and other indirect voice therapy approaches. This finding is consistent with that of Mathieson, Van Lierde et al., ${ }^{10,16}$ who reported positive effects in voice quality following laryngeal manual therapy. Similar positive effects using an eclectic voice therapy program was reported by Singh et al. ${ }^{23}$ in individuals diagnosed with MTD and also associated with nodules, polyp, presbylaryngis, laryngopharyngeal reflux, and cut throat injury in the age range of 20 to 70 years. The voice therapy included five aspects.

\section{a. programme on vocal hygiene}

b. symptomatic voice therapy (circumlaryngeal massage, chewing exercises, yawn sigh approach, phonation on inhalation)

c. Respiratory retraining (Breath support, confidential voice therapy),

d. physiologic voice therapy (vocal function exercises, accent method, resonant voice therapy

e. Psychogenic voice therapy. Another study by Rangarathnam et al., ${ }^{24}$ evaluated the effects of 10-15 minutes of vocal hygiene programme and 20-25minutes of airflow exercises through teletherapy mode in individuals with MTD. Positive effects were found in the perceptual and voice related quality-of-life outcomes.

The DSI value of twelve out of the twenty participants reached normal range of DSI of Kannada speakers ranging from 3.79 to 6.25. ${ }^{13,25}$ In case of remaining eight participants, although they improved from their baseline DSI, did not reach the reference value 
of DSI with six sessions of therapy. ${ }^{26-28}$ Further, the participants M, $\mathrm{Q}$, and $\mathrm{T}$ achieved normal DSI value by the end of two sessions; participants $\mathrm{E}, \mathrm{O}$, and $\mathrm{S}$ reached this value after four sessions; $\mathrm{B}, \mathrm{K}$, $\mathrm{L}, \mathrm{M}$, and $\mathrm{N}$ took six sessions to attain this level. The participants $\mathrm{C}$ and I however, were having their baseline DSI value itself within normal range. These participants however, continued voice therapy programme despite reaching the normal DSI value, as they were to achieve the ease of voice and to be normal in terms of perception. The variations in terms of the number of sessions taken to reach normalcy can be based on several vocal related and unrelated factors. The vocal related factors include their variations with respect to the vocal pathology and the degree of dysphonia at baseline. However, the non vocal factors such as motivation, practice, professional vocal demands, vocal loading could also have lead to the variation in the magnitude of prognosis.

\section{Conclusion}

The study attempted to investigate the efficacy of an eclectic voice therapy programme on voice quality in individuals with hyperfunctional voice disorders. It was hypothesized that an eclectic voice therapy program comprising of patient education along with systematic implementation of symptomatic voice therapy approaches could promote better prognosis in individuals with HFVD. In order to verify the hypothesis, 20 participants in the age range of 15-65years diagnosed with HFVD by a team consisting of a Laryngologist and a Speech Language Pathologist were considered for the study. Outcome of the therapy was documented systematically through a single subject time series design involving documentation of the objective parameter dysphonia severity index before commencing the therapy (baseline) and following every two sessions during the therapy. Despite the individual variations in terms of the magnitude of improvement, the DSI value of all the participants moved in positive direction indicating improved voice with proposed EVT program. The improvement in the DSI value with therapy can be attributed to the improvement in the respiratory capacity, respiratory-phonatory control, the relaxed laryngeal frame work and improved vocal stability. Therefore, it may be concluded that the proposed eclectic voice therapy programme can be considered as one of the efficient approaches in the management of individuals with hyperfunctional voice disorders.

\section{Acknowledgments}

None.

\section{Conflicts of interest}

Author declares there are no conflicts of interest.

\section{Funding}

None.

\section{References}

1. Verdolini K, Rosen CA, Branski RC.Classification manual for voice disorders-1. Mahwah, Lawrence Erlbaum Associates, NJ, USA. 2006.

2. Williams NR. Occupational groups at risk of voice disorders. In Williams N, Carding P, editors. Occupational Voice Loss. 1st ed. London. Boca Rator, Taylor Francis Group, UK. 2005. p. 11-12.

3. Smith E, Gray SD, Dove H, et al. Frequency and effects of teachers voice problems. $J$ Voice. 1997;11(1):81-87.

4. Smith E, Lemke J, Taylor M, et al. Voice problems among teachers: differences by gender and teaching characteristics. $J$ Voice. 1998;12(4):328-334.
5. Smith E, Kirchner HL, Taylor M, et al. Frequency of voice problems among teachers and other occupations. J Voice. 1998;12(3):480-488.

6. Sliwinska Kowalska M, Niebudek Bogusz E, Fiszer M, et al. The Prevalence and Risk Factors for Occupational Voice Disorders in Teachers. Folia Phoniatr Logop. 2006;58(2):85-101.

7. Sebastian S, Suresh BA, Simon S, et al. Risk Factors for Hyperfunctional Voice Disorders among Teachers. Online $J$ Health Allied Scs. 2012;11(2):1-3

8. Aronson AE. Clinical voice disorders. Thieme Medical, New York, USA. 1990.

9. Roy N, Bless DM, Heisey D, et al. Manual circumlaryngeal therapy for functional dysphonia: An evaluation of short- and long-term treatment outcomes. $J$ Voice. 1997;11(3):321-331.

10. Mathieson L, Hirani SP, Epstein R, et al. Laryngeal manual therapy: a preliminary study to examine its treatment effects in the management of muscle tension dysphonia. $J$ Voice. 2009;23(3):353-366.

11. Van Lierde KM, De Bodt M, Dhaeseleer E, et al. The Treatment of Muscle Tension Dysphonia: A Comparison of Two Treatment Techniques by Means of an Objective Multiparameter Approach. $J$ Voice. 2010;24(3):294-301.

12. Wuyts FL, De Bodt MS, Molenberghs G, et al. The Dysphonia Severity Index: An Objective Measure of Vocal Quality Based on a Multiparameter Approach. J Speech Lang Hear Res. 2000 43(3):796-809.

13. Jayakumar T, Savithri SR. Effect of geographical and ethnic variation on Dysphonia Severity Index. A study of Indian population J Voice. 2012;6 (1):e11-e16.

14. Van Lierde KM, Claeys S, De Bodt M,et al. Long-term outcome of hyperfunctional voice disorders based on a multiparameter approach. $J$ Voice. 2007;21(2):179-188.

15. De Bodt MS. A framework for voice assessment: The relation between subjective and objective parameters in the judgment of normal and pathological voice. Doctoral dissertation. University of Antwerp, Belgium. 1997.

16. Van Lierde KM, Claeys S, De Bodt M, et al. Vocal quality characteristics in children with cleft palate: A multiparameter approach. $J$ Voice. 2004;18(3):354-362.

17. Amir O, Dukas, M, Shnaps BaumR. The effect of a voice course on the voices of people with and without pathologies: preliminary observations. Logoped Phoniatr Vocol. 2003;30(2):63-71.

18. Wingate JM, Brown WS, Shrivastav R, et al. Treatment outcomes for professional voice users. $J$ Voice. 2007;21(4):433-449.

19. Roy N, Leeper HA. Effects of the manual laryngeal musculoskeletal tension reduction technique as a treatment for functional voice disorders: perceptual and acoustic measures. J Voice. 1993;7(3):242-249.

20. Pettersen V, Westgaard RH. Muscle activity in professional classical singing : a study on muscles in the shoulder, neck and trunk. Logoped Phoniatr Vocol. 2004;29(2):56-65.

21. Pettersen V, Westgaard RH. The activity patterns of neck muscles in professional classical singing. $J$ Voice. 2005;19(2):238-251.

22. Jenny Iwarsson. Breathing and Phonation-Effects of Lung Volume and Breathing Behaviour on Voice Function. Doctoral Dissertation from Dept. of Logopedics \& Phoniatrics, Karolinska Institute and Dept of Speech, Music \& Hearing, Royal Institute of Technology, Stockholm 2001. Stockholm, Sweden. 2001. p.121.

23. Singh SP, Dutta SRB. Voice Therapy in Muscle Tension Dysphonia Cases. International Journal of Phonosurgeryand Laryngology. 2015;5(1):20-24. 
24. Rangarathnam B, McCullough GH, Pickett H, RI, et al. Telepractice versus In-Person Delivery of Voice Therapy for Primary Muscle Tension Dysphonia. Am J Speech Lang Patho. 2015;24(3):386-399.

25. Baker J, Ben Tovim DI, Butcher A, et al. Development of a modified diagnostic classification system for voice disorders with inter-rater reliability study. Logoped Phoniatr Vocol. 2007;3(3):99-112.

26. Bassiouny S. Efficacy of the accent method of voice therapy. Folia Phoniatr Logop. 1998;50:146-164.
27. Boone DR, McFarlane SC. The voice and voice therapy (4th edn.). Englewood Cliffs, Prentice-Hall, New Jersey, USA. 1988.

28. Dromey C, Nissen SL, Roy N, et al. Articulatory changes following treatment of muscle tension dysphonia: preliminary evidence. $J$ Speech Lang Hear Res. 2008;51(1):196-208. 